\title{
VARIATIONS ON THOMPSON'S CHARACTER DEGREE THEOREM
}

\author{
GABRIEL NAVARRO \\ Departament d'Algebra, Facultat de Matematiques, Universitat de Valencia, \\ 46100 Burjassot, Valencia, Spain \\ email:gabriel@uv.es \\ and THOMAS WOLF \\ Department of Mathematics, Ohio University, Athens, OH 45701, USA \\ email:wolf@ohiou.edu
}

(Received 8 November, 2000; accepted 30 March 2001)

\begin{abstract}
If $P$ is a Sylow- $p$-subgroup of a finite $p$-solvable group $G$, we prove that $G^{\prime} \cap \mathbf{N}_{G}(P) \subseteq P$ if and only if $p$ divides the degree of every irreducible non-linear $p$-Brauer character of $\mathrm{G}$. More generally if $\pi$ is a set of primes containing $p$ and $G$ is $\pi$-separable, we give necessary and sufficient group theoretic conditions for the degree of every irreducible non-linear $p$-Brauer character to be divisible by some prime in $\pi$. This can also be applied to degrees of ordinary characters.
\end{abstract}

2000 Mathematics Subject Classification. 20C15.

A well-known Theorem of Thompson (12.2 of [3]) states that a finite group $G$ has a normal $p$-complement if the degree of every non-linear irreducible complex character of $G$ is divisible by $p$. Gow and Humphreys [1] proved that the same conclusion holds if $p$ divides the degree of every non-linear irreducible $q$-Brauer character for a prime $q \neq p$ when $G$ is $q$-solvable. They also showed that there exist $p$-solvable groups of arbitrary $p$-length, where $p$ divides the degree of every non-linear irreducible $p$-Brauer character. We use known results about the McKay conjecture to give necessary and sufficient conditions for $p$ to divide the degree of every non-linear irreducible $p$-Brauer character of a $p$-solvable group $G$.

THeOREM A. The degree of every non-linear p-Brauer character of a p-solvable group $G$ is divisible by $p$ if and only if $G^{\prime} \cap \mathbf{N}_{\mathrm{G}}(P) \subseteq P$ whenever $P$ is a Sylow-p-subgroup of $G$.

More generally, given a set of primes $\pi$ with $p \in \pi$, we give in Theorem B necessary and sufficient group-theoretic conditions for the degree of each non-linear irreducible $p$-Brauer character of a $\pi$-separable group $G$ to be divisible by some prime in $\pi$, at least when $G$ satisfies some separability conditions. This can also be applied to ordinary characters of $G$ by choosing $p$ with $(p,|G|)=1$.

Actually Theorem A is valid whenever $G$ satisfies McKay's conjecture for $p$ Brauer characters of $p^{\prime}$-degree. Theorem $\mathrm{A}$ is not true for all finite groups, at least 
when $p=2$. The simple group of order 168 has a self-normalizing Sylow-2-subgroup, but it also has two irreducible 2-Brauer characters of degree 3 . The other direction also fails in general since the simple group of order 60 does not have a self-normalizing Sylow-2subgroup, yet the degrees of its irreducible 2-Brauer characters are 1,2,2, and 4.

We conjecture that Theorem A is valid for all finite groups when $p$ is odd, but it seems hard to find groups $G$ satisfying either condition with $p<2$ and $G$ not $p$-solvable. An example of a group $G$ that is not $p$-solvable and satisfies both conditions with $p=3=\left|G: G^{\prime}\right|$ has $G^{\prime} \cong L_{2}(27)$. (See [4].) For $p>3$, a theorem of Glauberman shows that no Sylow-p-subgroup of a simple non-abelian group is self-normalizing. (See the Corollary to Theorem 5.14 of [7].)

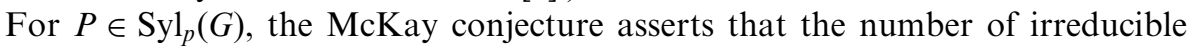
characters of $G$ with $p^{\prime}$-degree equals the number of such characters of $\mathbf{N}_{G}(P)$. For $p$ solvable groups, E. Dade first proved this, and a simpler proof due to Okuyama and Wajima appears in [5]. The following generalization is the main result of [8].

THeORem 1. If $G$ is p-solvable and $\pi$-separable for a set of primes $\pi$ and $H$ is a Hall- $\pi$-subgroup of $G$, then

$$
\mid\left\{\varphi \in \operatorname{IBr}_{p}(G) \mid \varphi(1)\right.
$$

is $a \pi^{\prime}$-number $\left.\mid\right\}=\mid\left\{\eta \in \operatorname{IBr}_{\mathrm{p}}\left(\mathrm{N}_{\mathrm{G}}(\mathrm{H})\right) \mid \alpha(1)\right.$ is a $\pi^{\prime}$-number $\mid$.

Theorem $\mathrm{A}$ is immediate from Theorem B by setting $\pi=\{p\}$.

Theorem B. Let $H$ be a Hall- $\pi$-subgroup of a $\pi$-separable group $G$ for a set $\pi$ of primes with $p \in \pi$. Assume that $G$ is p-solvable. Then the degree of every non-linear irreducible p-Brauer character of $G$ is divisible by some prime in $\pi$ if and only if $G^{\prime} \cap \mathbf{N}_{G}(H) / H^{\prime}$ is a p-group.

Proof. We first prove the theorem when $H$ is normal in $G$. Let $P \in S y l_{p}(G)$. Now $H^{\prime} P \subseteq H$ and $H^{\prime} P / H^{\prime}$ is a normal Sylow- $p$-subgroup of $G / H^{\prime}$. Since $H / H^{\prime} P$ is a normal abelian Hall- $\pi$-subgroup of the $p^{\prime}$-group $G / H^{\prime} \mathrm{P}$, the degree of every $\varphi \in \operatorname{Irr}\left(G / H^{\prime} P\right)=\operatorname{IBr}_{p}\left(G / H^{\prime} P\right)$ is a $\pi^{\prime}$-number. Now assume that the degree of every non-linear irreducible $p$-Brauer character of $G$ is divisible by some prime in $\pi$. Then every $\varphi \in \operatorname{Irr}\left(G / H^{\prime} P\right)$ is linear, from which it follows that $G^{\prime} \subseteq H^{\prime} P$ and that $G^{\prime} / H^{\prime}$ is a $p$-group. For the converse, assume that $G^{\prime} / H^{\prime}$ is a $p$-group. Then $G^{\prime} \subseteq H^{\prime} P$ and every $\varphi \in \operatorname{Irr}\left(G / H^{\prime} P\right)$ is linear. Since $H^{\prime} P / H^{\prime}$ is a $p$-group, every irreducible $p$-Brauer character of $G / H^{\prime}$ is linear. Consequently if $\beta$ is a non-linear irreducible $p$-Brauer character of $G$, then an irreducible constituent $\gamma$ of $\beta_{H^{\prime}}$ is not principal. We may choose $\alpha \in \operatorname{IBr}_{p}(H)$ that is an irreducible constituent of $\beta_{H}$ that lies over $\gamma$. By $p$-solvability, the degree of $\alpha$ divides $|\mathrm{H}|$. Also $\alpha$ is not linear because the irreducible constituents of $H^{\prime}$ are not principal. Hence $\alpha(1)$ is divisible by some prime in $\pi$. Furthermore $\alpha(1)$ divides $\beta(1)$ by Clifford's Theorem. (See [6, Corollary 8.7].) Hence $\beta(1)$ is divisible by some prime in $\pi$. This establishes the Theorem when $H$ is normal in $G$. Setting $N=\mathbf{N}_{G}(H)$, we may now assume that $N<G$.

Now $G^{\prime} H$ is a normal subgroup of $G$ of $\pi^{\prime}$-index, so that the Frattini argument implies that $G=\left(G^{\prime} H\right) N=G^{\prime} N$ and $G / G^{\prime} \cong N / G^{\prime} \cap N$. Let $J_{\pi^{\prime}}(G)$ denote the set of irreducible $p$-Brauer characters of $G$ of $\pi^{\prime}$-degree. Since $G$ is $p$-solvable and $\pi$-separable, Theorem 1 implies that $\left|J_{\pi^{\prime}}(G)\right|=\left|J_{\pi^{\prime}}(N)\right|$. Assume that all $\varphi \in J_{\pi^{\prime}}(G)$ are linear. Then $J_{\pi^{\prime}}(G)=\operatorname{IBr}_{p}\left(G / G^{\prime}\right)$. Since $G / G^{\prime} \cong N / N \cap G^{\prime}$ and $\left|J_{\pi^{\prime}}(G)\right|=\left|J_{\pi^{\prime}}(N)\right|$, it follows that $J_{\pi^{\prime}}(N)=\operatorname{IBr}_{p}\left(N / N \cap G^{\prime}\right)$. Thus every $\mu \in J_{\pi^{\prime}}(N)$ is linear and also 
$N \cap G^{\prime} / N^{\prime}$ must be a $p$-group. Since $H$ is normal in $N$, we have by the first paragraph, that $N^{\prime} / H^{\prime}$ is a $p$-group. Then $N \cap G^{\prime} / H^{\prime}$ is a $p$-group, as desired.

Conversely assume that $N \cap G^{\prime} / N^{\prime}$ is a $p$-group. Then $N^{\prime} H^{\prime}$ is a $p$-group and so every $\mu \in J_{\pi^{\prime}}(N)$ is linear, by the first paragraph. Now $N \cap G^{\prime} / N^{\prime}$ is a $p$-group and so $J_{\pi^{\prime}}(N)=\operatorname{IBr}_{p}\left(N / N \cap G^{\prime}\right)$. Since $G / G^{\prime} \cong N / N \cap G^{\prime}$ and $\left|J_{\pi^{\prime}}(G)\right|=\left|J_{\pi^{\prime}}(N)\right|$, we have $J_{\pi^{\prime}}(G)=I B r_{p}\left(G / G^{\prime}\right)$ and all $\varphi \in J_{\pi^{\prime}}(G)$ are linear, as desired.

We used $p$-solvability twice in the proof of Theorem $\mathrm{B}$, namely to conclude that $\left|J_{\pi^{\prime}}(G)\right|=\left|J_{\pi^{\prime}}(N)\right|$ via Theorem 1 and to assume that $\beta(1)|| H \mid$ for all $\beta \in\left(\operatorname{IBr}_{p}(H)\right.$.

If $G$ satisfies the conditions of Theorem $\mathrm{B}$, it follows from the Theorem that $\mathbf{N}_{G}(H)$ does too. But the converse is false, as is evidenced by a semi-direct product $G=E P$, where $E$ is an extra-special $r$-group for a prime $r \neq p, P$ has order $p$, and $\mathbf{C}_{E}(P)=\mathbf{Z}(E)$. Then $\mathbf{N}_{G}(P)^{\prime}=1$ and the degree of every non-linear irreducible $p$-Brauer character of $\mathbf{N}_{G}(P)$ is divisible by $p$ (vacuously), while $G$ has an irreducible $p$-Brauer character of degree $r$.

Corollary 1. Suppose that $G$ is p-solvable and the degree of every non-linear pBrauer character of $G$ is divisible by $p$. Let $K=\mathbf{O}^{p^{\prime}}(G)$ and $P \in \operatorname{Syl}_{p}(G)$.

(i) $G / K \cong \mathbf{N}_{G}(P) / N_{K}(P)$ is abelian.

(ii) The degree of every non-linear $p$-Brauer character of $K$ is divisible by $p$

(iii) $\mathbf{N}_{K}(P)=P$.

Proof. The Frattini argument shows that $G=K \mathbf{N}_{G}(P)$ and so $G / K \cong$ $\mathbf{N}_{G}(P) / \mathbf{N}_{K}(P)$. The hypotheses imply that every $\chi \in \operatorname{Irr}(G / K)=\operatorname{IBr}_{p}(G / K)$ is linear and thus $G / K$ is abelian. Applying Theorem A twice, we have that $K^{\prime} \cap \mathbf{N}_{K}(P) \subseteq$ $G^{\prime} \cap \mathbf{N}_{G}(P) \subseteq P$ and the degree of every non-linear $p$-Brauer character of $K$ is divisible by $p$.

Since $K$ has no non-trivial $p^{\prime}$-factor groups, it follows that $K^{\prime} P=K$ and hence that $\mathbf{N}_{K}(P) / P \cong K^{\prime} \cap \mathbf{N}_{K}(P) / K^{\prime} \cap P$. But $K^{\prime} \cap \mathbf{N}_{K}(P) \subseteq P$ by the last paragraph. Thus $\mathbf{N}_{K}(P) / P$ is both a $p$-group and a $p^{\prime}$-group, whence $\mathbf{N}_{K}(P)=P$.

That $G / K \cong \mathbf{N}_{G}(P) / \mathbf{N}_{K}(P)$ in part (i) above is solely a consequence of the Frattini argument. The conclusion above that $\mathbf{N}_{K}(P)=P$ thus shows that $\mathbf{N}_{G}(P)$ is as small as possible. Applying the classification of finite simple groups, we get the following result.

COROLlary 2. If the degree of every non-linear p-Brauer character of a p-solvable group $G$ is divisible by $p$, then $G$ is solvable.

Proof. Let $K=\mathbf{O}^{p^{\prime}}(G)$. By Corollary 1 , we have that $G / K$ is abelian and so we may choose a minimal normal subgroup $M$ of $G$ with $M \subseteq K$. Arguing by induction on $|G|$, we conclude that $G / M$ is solvable and so we may assume that $M$ is a $p^{\prime}-$ group. Now $\mathbf{C}_{M}(P) \subseteq \mathbf{N}_{K}(P)=P$ by Corollary 1 . Thus $M$ admits a coprime automorphism group $P$ with trivial centralizer and it is a well-known consequence of the classification of simple groups that $M$ must be solvable. Thus $G$ is solvable.

As sketched by the referee, there is a reasonably direct proof of Corollary 2 that bypasses Theorem A, but that still uses the classification. By choosing the prime $p$ to be coprime to $|G|$ in Theorem $\mathrm{B}$, we next get results about ordinary characters. 
COROLlary 3. Let $H$ be a Hall- $\pi$-subgroup of a $\pi$-separable group $G$. The degree of every non-linear $\chi \in \operatorname{Irr}(G)$ is divisible by some prime in $\pi$ if and only if $G^{\prime} \cap \mathbf{N}_{G}(H)=H^{\prime}$.

When $\pi$ is a singleton $\{q\}$ in Corollary 3 , the hypothesis that $G$ is $q$-solvable is unnecessary, because $G$ must be $q$-nilpotent by the character theoretic condition and Thompson's Theorem (12.2 of [3]) or by the group theoretic condition and Tates's Theorem (IV 4.7 of [2]). In Corollary 3, the hypothesis that $G$ is $\pi$-separable may be replaced by the hypothesis that the number of irreducible characters of $\mathrm{G}$ of $\pi^{\prime}$-degree and the number of such characters of $\mathbf{N}_{G}(H)$ are equal, as evidenced by remarks after Theorem B.

Thompson's Theorem (12.2 of [3]) on character degrees and Corollary 3 show that a prime $q$ divides the degree of every non-linear ordinary character of a finite group $G$ if and only if $G$ has a normal $q$-complement $M$ and $\mathbf{C}_{M^{\prime}}(Q)=1$ when $Q \in \operatorname{Syl}_{q}(G)$, an equivalence derived in [1]. This condition on character degrees not only implies $q$-solvability, but even solvability. (See the proof of Corollary 2.)

COROLlary 4. If $H$ is a Hall-p'-subgroup of a p-solvable group $G$, then $G^{\prime} \cap N_{G}(H)=H^{\prime}$ if and only if $G$ has no nonlinear irreducible character whose degree is a power of $p$.

\section{REFERENCES}

1. R. Gow and J. Humphreys, Normal p-complements and irreducible representations, J. London Math. Soc. (2) 11 (1975), 308-312.

2. B. Huppert, Endliche Gruppen I (Springer-Verlag, 1967).

3. I. M. Isaacs, Character theory of finite groups (Dover, New York, 1994).

4. C. Jantzen, K. Lux, R. Parker and R. Wilson, An atlas of Brauer characters, London Math. Soc. Monographs (Clarendon Press, Oxford, 1995).

5. O. Manz and T. Wolf, Representations of solvable groups (Cambridge University Press, 1993). 1998)

6. G. Navarro, Characters and blocks of finite groups (Cambridge University Press,

7. M. Suzuki, Group theory II (Springer Verlag, 1986). $123-138$

8. T. Wolf, Variations on McKay's character degree conjecture, J. Algebra 174 (1990), 